\title{
Adding ICT to Transportation Systems: Monitoring and Management System for the Electric Vehicle Fleet on the Island of Ventotene
}

\author{
G. Fabbri, C. Boccaletti, L. Anniballi, M. London, F. Calenne, F. M. F. Mascioli, and A. J. M. Cardoso
}

\begin{abstract}
This paper describes an Automatic Vehicle Monitoring and Location System developed to be tested on the municipal fleet of electric and hybrid vehicles circulating on the small island of Ventotene in Italy. The system allows to determine the geographic position and to provide data about the vehicles, and sends the information to a remote server.
\end{abstract}

Index Terms-Automatic vehicle monitoring and location systems, electric vehicles, fault tolerance, intelligent transportation systems.

\section{INTRODUCTION}

Transportation systems in Europe are in the early stage of being radically transformed by Information and Communications Technology (ICT). Adding ICT to the transport infrastructure, vehicles and transport management is slowly leading to the development of Intelligent Transport Systems (ITS), which include a wide and growing suite of technologies and applications [1]. These systems deal with several combinations of communication, computer and control technologies developed and applied in the domain of transport to improve system performance, transport safety, efficiency, productivity, level of service, environmental impact, energy consumption and mobility. Other benefits come from expanding economic and employment growth. The potential of ITS has encouraged the EU to make them an integral part of the Common Transport Policy [2], with the aim of establishing a coordinated infrastructure for ITS in Europe and to contribute to the development, assessment and demonstration of ITS applications, laying the groundwork for a large deployment of ITS in the future.

To classify ITS applications five primary categories can be identified:

1) Advanced Traveler Information Systems (ATIS);

2) Advanced Transportation Management Systems

Manuscript received September 2, 2012; revised November 16, 2012. This work was supported in part by the Portuguese Foundation for Science and Technology (FCT) under Project No. SFRH/BPD/46224/2008 and in part by the Pole for Sustainable Mobility.

G. Fabbri is with the Instituto de Telecomunicações , Department of Electrical and Computer Engineering, Pólo II - Pinhal de Marrocos, P3030-290, Coimbra, Portugal (e-mail: gianluca.fabbri@co.it.pt).

L. Anniballi, M. London, F. Calenne, and F. M. F. Mascioli are with the Department of Information, Electronic and Telecom Engineering, Sapienza University of Rome, Rome, Italy (e-mail: fabiana.calenne@pomos.it, marco.london@pomos.it, fabio.mascioli@pomos.it).

C. Boccaletti is with the Department of Astronautics, Electrical and Energetic Engineering, Sapienza University of Rome, Rome, Italy (e-mail: chiara.boccaletti@uniroma1.it).

A. J. M. Cardoso is with the Department of Electromechanical Engineering, University of Beira Interior, Covilhã, Portugal, and also with the Instituto de Telecomunicações (e-mail: ajmcardoso@ieee.org).
(ATMS);

3) ITS-Enabled Transportation Pricing Systems;

4) Advanced Public and Private Transportation Systems (APTS);

5) Fully Integrated ITS Systems (VII and V2V Systems).

This classification is not inclusive of all possible ITS applications but includes the most prominent ones arranged by their primary functional intent and considering that many ITS applications can serve multiple functions or purposes. Interesting applications in the APTS category are the Automatic Vehicle Monitoring (AVM) and Automatic Vehicle Location (AVL) systems [3]-[4]. These systems represent an electronic means of gathering data and performing commands over a land vehicle fleet. Since data on vehicle location are required for an effective control, such systems are totally dependent upon reliable dedicated communications systems [5]. This paper describe an AVL and AVM system developed to be tested on the small island of Ventotene in Italy, in order to determine the geographic position and provide information about a small municipal fleet of electric and hybrid vehicles circulating on the island and to send the information to a remote server.

\section{VEHICLE MONITORING AND LOCATION SYSTEMS}

The design and implementation of AVL and AVM systems includes the acquisition and transmission of the information about the vehicle's location and on-board devices status to a monitoring server. The main components of these systems are usually the following:

Remote devices. In order to track the location of a vehicle, some kind of device is needed in the remote location. It typically consists of a GPS receptor and communication equipment. These two devices may be directly connected or integrated into a single component.

Communication component. It allows the system to communicate with remote GPS devices using wireless media

Data acquisition component. It builds and decodes messages for the remote GPS devices, including location queries, location responses and GPS configuration. It uses the communication component to send and receive these messages.

Real-time tracking component. It acts as a client for the data acquisition component. It obtains the locations of the remote devices and processes them as needed. For example, it can be connected to Geographic Information Systems (GIS) visualization or route storage components.

GIS visualization component. It shows locations and routes on digital maps. 
Route analysis component. It offers some basic tracking queries, which can be performed on a route, and a graphical user interface (GUI) to build more complex tracking queries.

Persistence component. All the above components and applications need to store the locations they receive or generate for further analysis. Usually, this data persistence is provided by a data base manager.

These components can be combined in order to build different systems. They should be able to solve different kinds of problems and act as distributed GPS data collectors, local area control centers or headquarters control, or provide distributed management of the same GPS data. As a general example Fig. 1 illustrates the architecture of the system presented in this work.

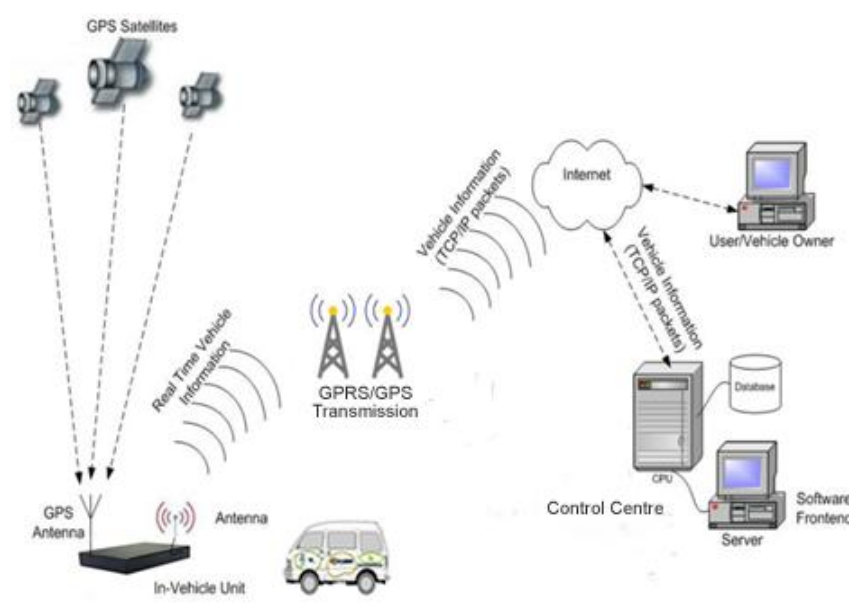

Fig. 1. System architecture.

\section{THE VHEICLE FLEET IN VENTOTENE}

The island of Ventotene is located in the Tyrrhenian Sea, and is part of the Pontine Islands in the region of Lazio, Italy (see Fig. 2). The island has an area of $1.247 \mathrm{~km}^{2}$ with a maximum length of $2.7 \mathrm{~km}$ and a maximum width of about $800 \mathrm{~m}$ and has about 700 permanent residents. During summertime, this number increases up to about 2500 due to the fact that many tourists each year choose the island as the venue for their holidays, thus resulting in a considerable increase in energy and mobility needs.

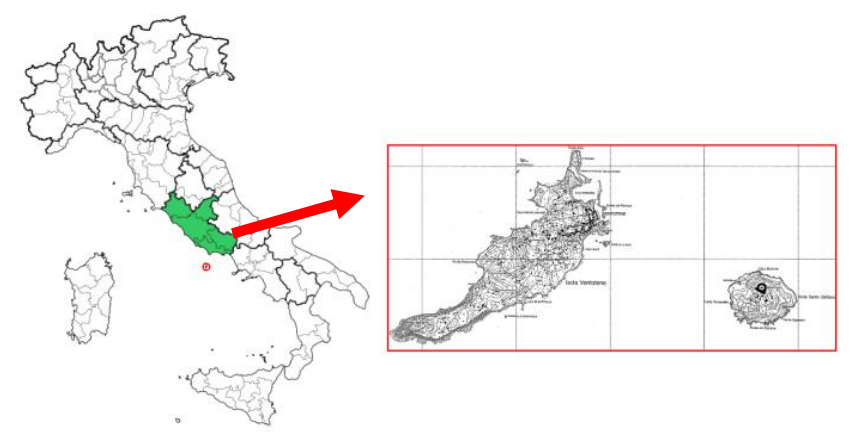

Fig. 2. The island of Ventotene.

The island is not connected to the electric grid on the mainland, and electricity is locally produced by means of a diesel power plant, with a considerable impact on the environment (i.e. pollutant emissions, noise and oil transport) and high generation costs. For this reason, the Municipality of Ventotene participated in a project named "Ventotene
Zero Emission Island" aimed at supporting the integration of Renewable Energy Sources (RES) as well as Sustainable Mobility actions in the island [6], trying to improve the citizens' trust by increasing the number of electric vehicles and system infrastructures through pilot projects. The project started in summer 2010 with the installation of the main infrastructures and the arrival on the island of the first electric vehicles to be used by the Municipality. The latter are 7 commercial Piaggio Porters using a pure electric drivetrain which only consumes electric energy. They are equipped with a $10.5 \mathrm{~kW}$ DC electric motor and 14 heavy-duty lead-acid battery packs $(6 \mathrm{~V}-180 \mathrm{~A})$.

The project has been financed by the Lazio regional government with the participation of the Municipality of Ventotene and has been developed by the Pole for Sustainable Mobility (POMOS) and partners. Charging stations have been installed at selected sites on the island to create a small Intelligent Networked Charging Infrastructure. The charging stations keep track of charging times and other data to allow remote monitoring of their utilization and correct functioning. A first integrated $1.92 \mathrm{kWp} \mathrm{PV} \mathrm{station}$ has been designed, built and installed for charging the electric vehicles and other two similar stations will be installed in the island. Fig. 3 shows the first installed charging station integrated with the PV generator. Assuming an average autonomy for the van of $112 \mathrm{~km}$ between recharges, the installation is able to recharge the daily operation of 3 vans allowing for each of them an average daily traveled distance of about $15 \mathrm{~km}$.

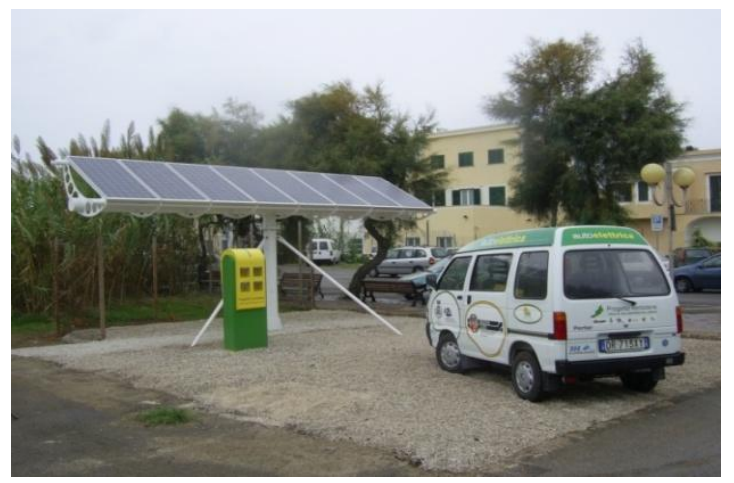

Fig. 3. The first PV integrated charging station installed on the island.

The first months of the project have shown that electric vehicles are very appropriate for use on the island, for both daily transport from the port to the centre and small trips around the island. All the experimental results and data coming from the charging infrastructure can be be used to learn more about what is needed to support electric vehicles as they become more and more common on the island. The increased use of electric vehicles will impact electric utilities and the infrastructure for providing electricity to customers. A further key element of the project was the design and implementation of an AVL and AVM system to monitor the small municipal fleet. The system will be described in the following paragraphs.

\section{DESCRIPTION OF THE SYSTEM}

The system consists of three subsystems: the Control Centre, the On-board Device and the Communication 
Network.

The Control Centre can be considered the main element of the whole system having the general function of collect and integrate all monitoring and localization data coming from the On-board Devices. Through the Internet Protocol (TCP/IP), data sent from the devices can be elaborated by the main server located at POMOS.

The On-board Device detects and sends data concerning the vehicle. Its main architecture consists of the following elements:

1) Communication and transmission GPRS module from and to the Control Centre (Telit GM862);

2) GPS localization antenna (SiRFstarIII);

3) PLC microcontroller with digital/analogical input (Arduino MEGA). The PLC can detect the vehicle ID, the latitude and longitude, electrical signals (voltages and currents), vehicle inclination, battery state of charge (SOC) and other signals coming from various sensors;

4) Serial TTL converter;

5) Firmware for the management of the hardware components;

6) Management software. The software is used for the connection with the provider; it can sample, pack and send the data.

The Communication Network uses the General Packet Radio Service (GPRS) to transfer data. As known, the main benefits of GPRS are that it reserves radio resources only when there is data to send and it reduces reliance on traditional circuit-switched network elements. The increased functionality of GPRS will decrease the incremental cost to provide data service improving the quality of data services as measured in terms of reliability, response time, and features supported.

Fig. 4 shows the main hardware components of the On-board Device, listed in Table I.

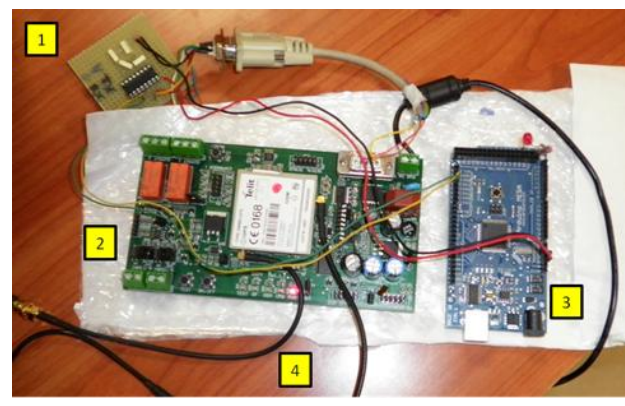

Fig. 4. The hardware of the on-board device.

TABLE I: DESCRIPTION OF THE HARDWARE.

\begin{tabular}{|l|l|l|}
\hline $\mathbf{N}^{\circ}$ & Description & Model \\
\hline 1 & Serial TTL Converter & Customized \\
\hline 2 & GPRS Module with GSM/GPRS antenna & TELIT GM862 \\
\hline 3 & PLC microcontroller & Arduino MEGA \\
\hline 4 & GPS Antenna & SiRFstarIII \\
\hline
\end{tabular}

The serial TTL converter has been designed to allow the logical communication between the microcontroller and the GPRS module. It also performs the voltage regulation (the Telit requires a $3.3 \mathrm{~V}$ voltage and the Arduino a $9 \mathrm{~V}$ voltage). The Telit GM862 has been chosen because it incorporates both the functions of localization and sending data combining GSM/GPRS and GPS. The module requires the use of two different antennas, one for the GSM/GPRS and the other one for the GPS signal (SiRFstarIII) and has an integrated SIM card holder. The transmission working frequencies in GSM mode range from 824.2 to $848.8 \mathrm{MHz}$ while the receiving frequencies range from 869.2 to $893.8 \mathrm{MHz}$. The transmitter nominal output power at $50 \mathrm{Ohms}$ is $2 \mathrm{~W}$ peak RF while the receiver sensitivity in typical operation conditions is equal to $-107 \mathrm{dBm}$. The GPS antenna has a $-159 \mathrm{dBm}$ internal sensitivity and provides the real time positioning of the vehicle with a position resolution accuracy of less than $2.5 \mathrm{~m}$. The position value is provided in the NMEA0183 output format string; this is a communication standard mainly used in marine and GPS satellite data communication which takes into account the time, the latitude, the longitude, the altitude, the speed and the date. The Arduino Mega can process various analogical and digital input signals, manage a GPS receiver, create data packs and control a modem. It is based on the AT Mega 1280 microcontroller and has $54 \mathrm{I} / \mathrm{O}$ lines with the possibility to generate 14 PWM signals, 16 analog inputs and 4 serial inputs and has a simple Wiring library that allows to program it in $\mathrm{C}$ and $\mathrm{C}++$. A series of sensors are connected to the microcontroller allowing to perform current and voltage measurements, to detect the temperature in various points of the vehicle, to analyze the quality of the air and to identify the inclination of the vehicle. Energy flow regulation must be a controlled process under any operating condition, which also requires a continuous measurement of current and voltage. Shunt resistors have been used to measure the voltage of the whole battery pack and of every single cell. Both the charging current balance and the exact battery voltage must be measured for billing purposes and to determine the battery's State Of Charge (SOC). To detect the residual energy in the batteries a Hall effect sensor, model LEM LA 55-P/SP1, has been used. Moreover, precision centigrade temperature sensors (model National Semiconductor LM35DZ) have been used to measure the temperature in the batteries. They are precision integrated-circuit temperature sensors, which output voltage is linearly proportional to the temperature in Celsius. The $\mathrm{LM} 35 \mathrm{DZ}$ is rated to operate over a $-55^{\circ}$ to $+150^{\circ} \mathrm{C}$ temperature range and draws only $60 \mu \mathrm{A}$ from its supply. The detection of Carbon Monoxide is possible thanks to the use of a Figaro TGS 2442 multilayer sensor. This sensor displays good selectivity to carbon monoxide, making it ideal for $\mathrm{CO}$ monitors. In the presence of $\mathrm{CO}$, the sensor's conductivity increases depending on the gas concentration in the air. A simple pulsed electrical circuit operating on a one second circuit voltage cycle can convert the change in conductivity to an output signal which corresponds to gas concentration. Fig. 5 shows typical sensitivity characteristics.

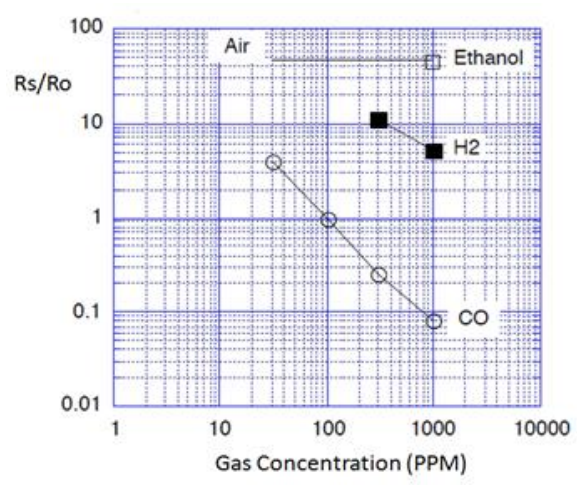

Fig. 5. Sensitivity characteristics of the temperature sensor. 
The $\mathrm{Y}$-axis is indicated as sensor resistance ratio (Rs/Ro) where $\mathrm{Rs}$ is the sensor resistance of displayed gases at various concentrations and Ro is the sensor resistance in 100 ppm CO. Motion, tilt and slope measurements are guaranteed by the use of $\mathrm{a} \pm 3 \mathrm{~g}$ tri-axis accelerometer. This is a Dimension Engineer ACCM3D model that presents integrated op amp buffers for the direct connection to the microcontroller's analog inputs. This sensor has a sensitivity up to $360 \mathrm{mV} / \mathrm{g}$, a $500 \mathrm{~Hz}$ bandwidth, operating voltage from $3.5 \mathrm{~V}$ to $15 \mathrm{~V}$ and draws $0.9 \mathrm{~mA}$.

\section{DATA Monitoring AND MANAGEMENT}

The direct connection of the sensors to the microcontroller and to the diagnostic bus can allow the automatic collection of vehicle performance data to support preventive maintenance and remote vehicle's diagnostics that can be provided through an on-board diagnostic system, which helps in controlling the vehicle (e.g. doors, motor, batteries and other possible systems) from a remote location. The On-board Device is empowered by a software application that manages the processes of local data acquisition and transmission of the acquired data to the remote server via GPRS. All data coming from the On-board Device are characterized by an ID to identify each single vehicle and are collected in a database to be used for statistical and graphical analysis and to set various alarms on the state of the vehicle, monitoring the operation and organizing the maintenance. A first prototype system has been designed and implemented at POMOS laboratories. The system has been tested on different electric and hybrid prototypes available at POMOS. Initial experiments and measurements have demonstrated the robustness, efficiency and applicability of the proposed system to the fleet in Ventotene. As an example, Fig. 6 shows the results obtained by measuring the battery current and the temperature during a phase of acceleration and deceleration of a $45 \mathrm{~kW}$ Brushless DC Motor Drive.

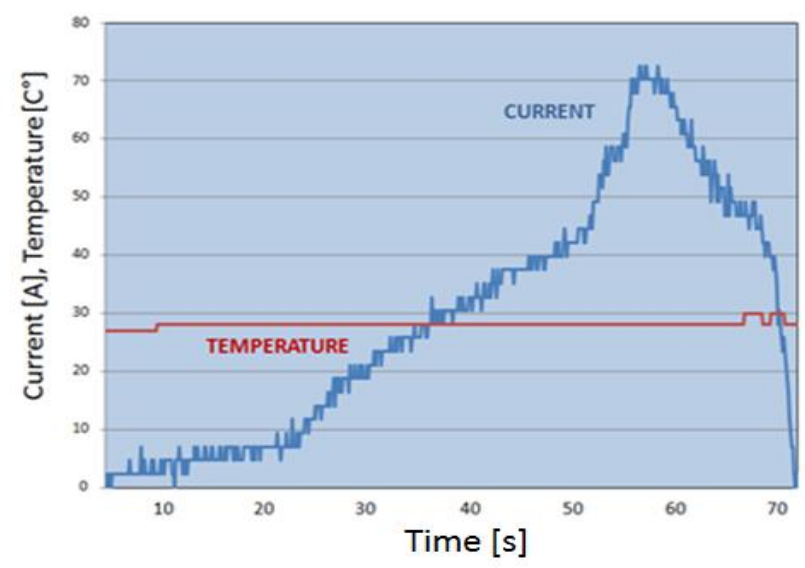

Fig. 6. Battery current and temperature during acceleration and deceleration of a brushless DC motor.

The On-board Devices will be mounted and tested on all the municipal electric vehicles during summer 2011 and on road experiments will be performed on the seven Vans. The system will be also tested on a selection of other commercial electric and hybrid vehicles and prototypes developed by POMOS together with different kind of batteries. All information about the vehicles will be remotely monitored by the Control Centre. Fig. 7 shows the web-based interface developed to display all transmitted information to the end user along with the location of the vehicle on a map. Fig. 8 shows the real time graphical location of a vehicle moving on the two main roads of the island. All the information can be made available to authorized users via a website. The system will be used to monitor the on-line performance and for service planning, safety and security, vehicle component monitoring, and data collection for future utilization.

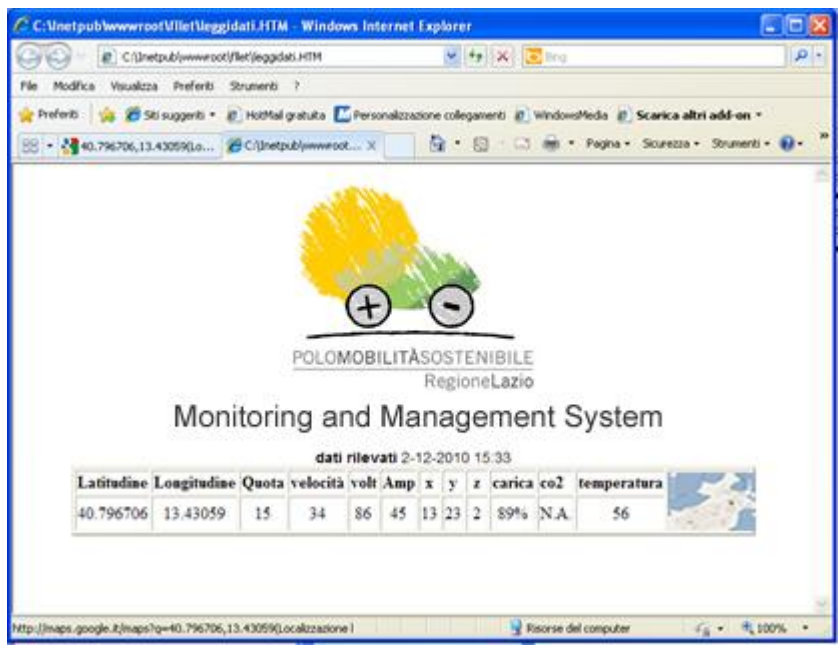

Fig. 7. Monitoring web based interface.

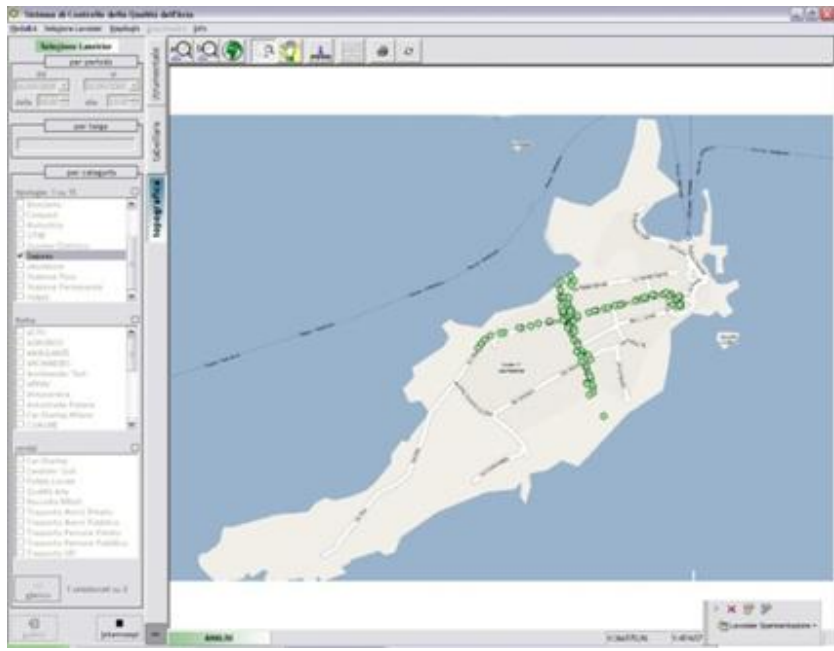

Fig. 8. Real time graphical location of a vehicle moving on the island.

Experimentation is continuing in summer 2011 also introducing other prototipal electric and hybrid vehicles in the fleet. Great importance will be given to identify possible fault tolerance strategies. The objective is to find solutions that provide fault tolerance to the most frequent faults, thereby, reducing the costs of dealing with the faults. The use of other kind of sensors will be also considered.

\section{CONCLUSIONS}

The first results of the project have shown that introducing ITS technologies for the monitoring and location of a fleet of vehicles can be an optimal solution to improve system performance, transport safety, efficiency, productivity, level of service, energy consumption and costs for a Municipality. The project experienced various technical and institutional problems. It can be observed that the biggest challenge in 
implementing AVL and AVM today is the potentially lengthy procurement and installation period (in particular, software development and integration of technical components). For this reason, public or private subjects procuring such a system may want to use an existing design, with customization capabilities, instead of developing a dedicated one, since such an approach would substantially limit potential risks and problems. It is also important to consider that the institutional relationships may be difficult and could slow down the progress of the projects. Another aspect that emerged from the analysis concerns the system operation: in order to correctly use the innovative components, new technical expertise is usually required and some existing staff might be reluctant to learn the new technology. A training program for the staff has to be considered in the programs and it is essential to exploit all the potential of the systems. As usual, the cost is the major concern: the cost of the development and of the installation of AVL and other advanced transportation system components is dependent on the size of the system, its level of sophistication, and the components to be included. Systems can range from those with fairly basic features (GPS, computer-assisted dispatching, mobile data terminals, silent alarms, and limited automated passenger information) to very comprehensive systems like the one here proposed. There is a significant cost for the equipment and software that reside at the operations/dispatch center. The per-vehicle cost of large fleets is less than for smaller fleets, assuming similar features, because the cost of this major infrastructure is distributed over a larger number of vehicles. Notwithstanding the good results of the project, (the project has been selected by the PRESS4TRANSPORT EU platform [7] as a pilot demonstration) the continuation of the experimentation is strictly dependent on the decision of the regional Municipality. In order to accelerate and stimulate the diffusion of ITS in Europe a public financing is indispensable.

\section{ACKNOWLEDGMENT}

This work was supported in part by the Portuguese Foundation for Science and Technology (FCT) under Project No. SFRH/BPD/46224/2008 and in part by the Pole for Sustainable Mobility.

\section{REFERENCES}

[1] S. Ezell, "Intelligent transportation systems," The Information Technology and Innovation Foundation, January 2010.

[2] E. Commission, "European transport policy for 2010: time to decide," White Paper, COM, CEC, Brussels, 2001, vol. 370.

[3] Intelligent Transportation Systems: Automatic Vehicle Location and Monitoring Systems. Thematic Fiches from the Press 4 Transport Consortium. June 2010. [Online]. Available: http: www.press4transport.eu.

[4] S. Riter and J. McCoy, "Automatic vehicle location: An overview," IEEE Trans. on Vehicular Technology, vol. 26, no. 1, pp. 7-11, 1977.

[5] M. A. A. Taee, O. B. Khader, and N. A. A. Saber, "Remote monitoring of vehicle diagnostics and location using a smart box with global positioning system and general packet radio service," in Proc. ACS/IEEE International Conference on Computer Systems and Applications, Amman, Jordan, May 13-16, 2007, pp. 385-388.

[6] F. Calenne, G. Fabbri, F. M. F. Mascioli, and S. Valentini, "Sustainable mobility models for the island of Ventotene," The International Multi-Conference on Complexity, Informatics and Cybernetics: IMCIC 2010, Orlando, Florida, USA, April 6-9, 2010.
[7] Virtual Press Office to improve EU Sustainable Surface Transport research media visibility on a national and regional level. [Online] Available: http://www.press4transport.eu

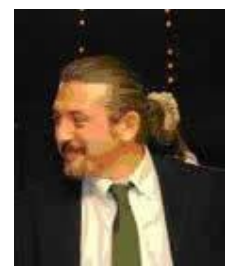

Gianluca Fabbri holds a Laurea degree in Electrical Engineering ("Sapienza" University of Rome) and a double European $\mathrm{PhD}$ in Electrical Engineering ("Sapienza" University of Rome and Madrid Polytechnic University). His research interests mainly regard Sustainable Mobility, components for stationary applications and simulation of Stand Alone Power Systems.

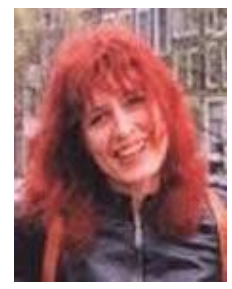

C. Boccaletti (M'04) was born in Bologna (Italy) in 1967. She received the Laurea degree in Mechanical Engineering and the $\mathrm{PhD}$ degree in Energetics from Sapienza University of Rome (Italy) in 1991 and 1995, repectively. In 1998 she was the recipient of the National Award "Energy and Environment" for her $\mathrm{PhD}$ thesis. She is an assistant professor at the Department of Astronautics, Electrical and Energetic Engineering of the Sapienza University of Rome. Her current research interests include design, analysis and optimisation of electrical machines, condition monitoring and diagnostics of electrical machines and drives, and systems based on renewable energies. She is co-author of two books and author of about 80 published papers in technical journals and conference proceedings.

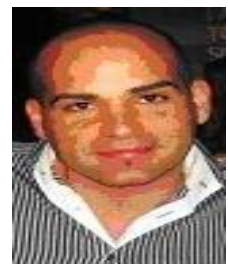

Marco London holds a degree in, Telecommunication Engineering ("Sapienza" University of Rome). Actually he is a PhD Student in Information and Communication Engineering at the Pole for Sustainable Mobility. His main research interests are Infomobility, Vehicular Ad Hoc Network (VANET), and intermodal integration of local systems for sustainable mobility.

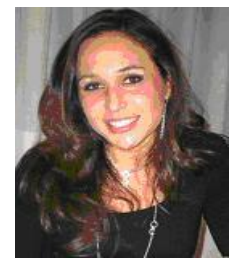

Fabiana Calenne holds a degree in Telecommunication Engineering ("Sapienza" University of Rome). Actually she is a PhD Student in Information and Communication Engineering at the Pole for Sustainable Mobility. His main research interests are Infomobility, Electromagnetic Compatibility and intermodal integration of territorial systems for sustainable mobility.

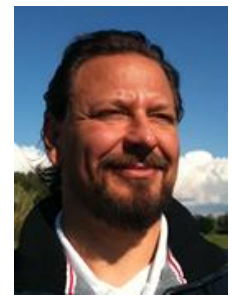

Fabio Massimo Frattale Mascioli was born in Rome, Italy, on June 13, 1963. He received the Laurea degree in Electronic Engineering in 1989 and the $\mathrm{PhD}$ degree in Information and Communication Engineering in 1995 from "Sapienza" University of Rome. He is Full Professor at the Department of Information, Electronic and Telecom Engineering of "Sapienza" University of Rome. His research interest mainly regards neural networks and neuro-fuzzy systems. Currently, he is also working on circuit modelling for vibration damping, energy conversion systems, electric and hybrid vehicles, energy-mobility integrated systems. He is author or coauthor of more than 80 papers published in technical journals and conference proceedings. From 2007, he is the scientific director of the Pole for Sustainable Mobility.

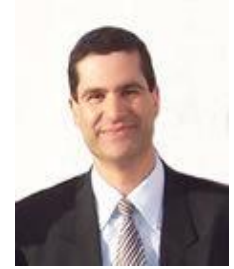

A. J. Marques Cardoso (S'89, A'95, SM'99) was born in Coimbra, Portugal, in 1962. He received the E. E. diploma, the Dr. Eng. degree and the Habilitation degree, all from the University of Coimbra, Coimbra, Portugal, in 1985, 1995 and 2008, respectively. From 1985 until 2011 he was with the University of Coimbra, where he was Director of the Electrical Machines Laboratory. Since 2011 he has been with the University of Beira Interior (UBI), Covilhã, Portugal, where he is a Full Professor at the Department of Electromechanical Engineering. His research interests are focused on condition monitoring and diagnostics of electrical machines and drives. He is the author of a book entitled Fault Diagnosis in Three-Phase Induction Motors (Coimbra, Portugal: Coimbra Editora, 1991), (in Portuguese) and more than 250 papers published in technical journals and conference proceedings. 